\title{
Oxidant Stress Responses in Premature Infants during Exposure to Hyperoxia
}

\author{
CHARLES V. SMITH, THOMAS N. HANSEN, NANCY E. MARTIN, HARILYN W. MCMICKEN, \\ AND STEPHEN J. ELLIOTT \\ Section of Neonatology: Department of Pediatrics and Department of Cell Biology: Baylor College of Medicine, \\ Houston, Texas 77030
}

\begin{abstract}
To assess oxidant stress responses in newborn infants treated with elevated concentrations of oxygen, we measured plasma concentrations of glutathione (GSH) and glutathione disulfide (GSSG) in newborn infants ranging from 23 to $\mathbf{4 2}$ wk gestational age. All infants recruited into the study were mechanically ventilated and had catheters placed in their umbilical arteries as part of their normal clinical management. Blood samples were obtained on $d 1,3$, and 5 and weekly thereafter or until the catheters were removed. We observed plasma concentrations of GSSG in these infants that were frequently an order of magnitude higher than the 0.1 to $0.3 \mu \mathrm{M}$ we find in adults. Interestingly, plasma GSSG concentrations were inversely correlated to the inspired oxygen tensions. This effect appeared to arise from the patient selection criteria whereby, of the infants studied, those breathing the lowest partial pressures of oxygen were the smallest and gestationally youngest. A second observation was that plasma concentrations of GSH in the premature infants were substantially, indeed often dramatically, lower than we have observed in adult humans (6 to $10 \mu \mathrm{M}$ ). Finally, we found that in patients with both umbilical arterial and umbilical venous catheters arterial GSSG concentrations were consistently higher than venous concentrations; conversely, arterial GSH concentrations were lower than venous concentrations. The elevated GSSG concentrations we observed in these infants indicate marked oxidant stress responses in prematurely born infants, even in those infants exposed only to room air. The positive arteriovenous gradients of GSSG concentrations across the lungs of these infants suggest that at least some of the increased plasma GSSG originates in the lung. The low plasma GSH concentrations we observed in these same infants suggest deficiencies in an antioxidant that has been shown in numerous animal studies to be critical for prevention of hyperoxia-induced lung injury. Finally, the negative arteriovenous gradients of GSH concentrations across the lung provide the first evidence in humans for pulmonary uptake of GSH. (Pediatr Res 34: 360-365, 1993)
\end{abstract}

\section{Abbreviations}

$\mathrm{FiO}_{2}$, fraction inspired oxygen

GGT, $\gamma$-glutamyltranspeptidase

GSH, glutathione

GSSG, glutathione disulfide

$\mathrm{PAO}_{2}$, alveolar oxygen tension

$\mathrm{PaO}_{2}$, arterial oxygen tension

Received January 26, 1993; accepted April 28, 1993

Correspondence and reprint requests: Charles V. Smith, Ph.D. Department of

Pediatrics, Baylor College of Medicine, One Baylor Plaza, Houston. TX 77030.

Supported by National Institutes of Health, PO1 GM34120, and the March of Dimes, Birth Defects Foundation.
The use of oxygen in the treatment of the newborn infant with respiratory insufficiency has both benefits and risks. Although often necessary to sustain life, increased concentrations of oxygen are toxic to cells and therapy with oxygen may play a role in the pathogenesis of chronic lung disease. Currently, no useful marker is available for the assessment of the magnitude of the oxidant stress imposed by hyperoxic therapy.

In recent years, the measurement of GSSG has been used in vitro and in vivo in experimental animals as a method of quantifying oxidant stress (1-5). In biologic systems, GSSG is formed largely through the enzymatic reduction of hydroperoxides by GSH. Under normal circumstances, most GSSG formed intracellularly is reduced back to GSH by glutathione reductase at the expense of NADPH oxidation, but many cells export GSSG to extracellular fluids such as plasma, lymph, or, in the case of the liver, bile (5).

Lambs and rats exposed to elevated oxygen concentrations have increased concentrations of GSSG in lung tissue $(4,6,7)$ In addition, administration of the redox-cycling lung toxin paraquat to rats has been shown to increase concentrations of GSSG in lung tissue and in blood plasma, particularly in arterial plasma (1). Therefore, the present studies were designed to determine whether GSSG is measurable in arterial plasma of newborn infants and to test the hypothesis that in newborn infants exposure to increased concentrations of oxygen, as part of their normal clinical management, would result in increased plasma concentrations of GSSG. The answers to both questions are important to clinicians because the ability to monitor oxidant stress responses in patient populations would be helpful in adjusting therapy and could offer clues about the mechanisms of adverse effects of hyperoxia exposure (8).

\section{MATERIALS AND METHODS}

Patient population. The patient population consisted of term and preterm infants admitted to the neonatal intensive care unit at Texas Children's Hospital between 1988 and 1990. After obtaining informed consent from the parents, we enrolled infants that met the entry criteria for the study. All infants were receiving mechanical ventilation and had umbilical artery catheters with their tips at the level of the third lumbar interspace (many of these patients also had umbilical venous catheters in place with their tips in the right atrium). The protocol was approved by the institutional review boards of Baylor College of Medicine and Texas Children's Hospital.

Clinical data. In addition to biochemical measurements, we maintained a data base of clinical data including birth weight, gestational age of birth, and admitting diagnoses. We recorded $\mathrm{PaO}_{2}$ and all data regarding ventilator settings including $\mathrm{FiO}_{2}$ and calculated $\mathrm{PAO}_{2}$. Blood samples were not drawn specifically for the measurement of arterial blood gas tensions at the time of sampling for the measurements of GSH and GSSG. The values 
used in the present data analyses are those obtained immediately before blood sampling. The time between sampling for clinical evaluation and experimental sampling was typically short, but the present method of data evaluation does not take into account possible interval-dependent effects.

Blood sampling and measurement of GSH and GSSG. After enrolling the infant in the study, we withdrew $1 \mathrm{~mL}$ of blood from the umbilical artery catheter. From those infants who also had umbilical venous catheters in place, we obtained a simultaneous sample of venous blood. We obtained subsequent $1-\mathrm{mL}$ samples on $\mathrm{d} 3$ and 5, and then weekly as long as the catheters were in place. Typically, we obtained the samples between 0800 and $1000 \mathrm{~h}$ and processed them immediately to avoid variations arising from possible diurnal rhythms in the infants and to minimize artifacts in GSH and GSSG measurements. Briefly, we added $0.25 \mathrm{~mL}$ of blood to an equal volume of ice-cold $50 \mathrm{mM}$ serine borate, $50 \mathrm{mM}$ potassium phosphate buffer containing $17.5 \mathrm{mM}$ EDTA as an anticoagulant, $\mathrm{pH} 7.4$, and gently mixed the sample by capping and inverting the microcentrifuge tube three times. Separately, we added $0.25 \mathrm{~mL}$ of blood to an equal volume of serine borate phosphate buffer containing $10 \mathrm{mM} \mathrm{N}$ ethylmaleimide and mixed it similarly. Then we centrifuged the samples immediately, separated the plasma samples, and analyzed them at that time or froze them at $-70^{\circ} \mathrm{C}$. We measured GSH and GSSG concentrations in plasma according to the method of Tietze adapted from the methods described by Adams et al. (1). In control experiments with adult human venous blood, we found no measurable change in plasma GSSG content with freezing at $-70^{\circ} \mathrm{C}$. Plasma GSH content decreased $10 \%$ after 1 $\mathrm{d}$ of freezing but then remained stable for $2 \mathrm{wk}$ after the first day.

Data analysis. We prospectively divided the infants enrolled in the study into three groups, according to birth wt and oxygen requirement.

Group 1 consisted of preterm infants of birth wt less than 1.5 $\mathrm{kg}$ who were intubated in the delivery room and ventilated in the first week of life to prevent apnea. These infants did not have lung disease and were usually ventilated with room air. No infant was included in this group if more than $40 \%$ oxygen was required. This group was considered at low risk for oxygen-induced lung injury and constituted the control population for exposure to hyperoxia.

Group 2 consisted of infants of all birth weights who were mechanically ventilated with elevated concentrations of oxygen for respiratory insufficiency secondary to parenchymal lung disease. This group consisted primarily of preterm infants with hyaline membrane disease and of developmentally more mature infants with parenchymal lung diseases, such as meconium aspiration pneumonia, bacterial pneumonia, or congenital heart disease and pulmonary edema. These infants were generally ventilated with high concentrations of oxygen and were considered at risk for development of oxygen-induced lung injury.

Group 3 consisted of near-term infants who were mechanically ventilated for respiratory failure without parenchymal lung disease. These infants all had idiopathic persistent pulmonary hypertension of the newborn and were intensely cyanotic despite having relatively normal chest $x$-rays and lung mechanics. They were an important control group because, in contrast to the infants in group 2 , they had no detectable parenchymal disease initially but were ventilated with nearly $100 \%$ oxygen for 3 to $7 \mathrm{~d}$.

Statistical analyses. We compared data among groups of patients using a one-way analysis of variance followed by a Newman-Keuls multiple comparison test, with significance at $p<$ 0.05 . Arteriovenous differences in GSH and GSSG concentrations were evaluated by paired $t$ tests. In addition, plasma concentrations of GSH and GSSG were compared with other variables by linear regression analyses (9).

\section{RESULTS}

Patient population. As expected, the infants in groups 1 and 2 were smaller and less mature than those in group 3 (Table 1). Infants in group 1 also required the least amount of supplemental oxygen (less than half that of group 2 or 3 ) and had the lowest alveolar oxygen tensions (Table 2). Arterial oxygen tensions were not different among the three groups.

Plasma GSSG concentrations. Plasma GSSG concentrations were $30 \%$ greater in group 1 infants compared with group 2 infants and almost twice those of group 3 infants. Least squares linear regression analysis of plasma GSSG concentrations versus $\mathrm{FiO}_{2}$ gave a line with a slope that was different from $0(p<$ 0.003 ) (Fig. 1). Unexpectedly, the slope of this line was -0.36 $\mu \mathrm{M}$ GSSG/atmosphere oxygen, suggesting that plasma GSSG concentrations were inversely related to $\mathrm{FiO}_{2}$. However, almost all infants in the low $\mathrm{FiO}_{2}$ group were members of group 1 and therefore were very immature. Thus, we performed a linear regression analysis of GSSG concentrations against gestational age. This analysis (Fig. 2) also gave a line with a slope that was different from $0, p<0.03$ (slope $=-0.016 \mu \mathrm{M}$ GSSG $/$ wk gestation).

The somewhat weaker (and negative) association of arterial plasma GSSG with gestational age indicated by linear regression analysis might be influenced by the restrictive and unnecessary assumption that a possible relationship be linear. Therefore, we compared plasma GSSG concentrations in infants born at less than $34 \mathrm{wk}$ of gestational age with those born at $34 \mathrm{wk}$ or more (Table 3 ). We found that arterial plasma GSSG concentrations were higher in the young infants $(p<0.008)$ than in the older infants (unpaired $t$ test), despite the significantly lower $\mathrm{FiO}_{2}$ and $\mathrm{PAO}_{2}$ values recorded for the younger infants.

We also obtained 66 simultancous measurements of GSSG concentrations in arterial and venous plasma (Table 4). Arterial GSSG concentrations were roughly $25 \%$ higher than in the corresponding venous samples. By paired $t$ test, this difference was significant for the group as a whole $(p<0.01)$ and for the subset of infants between 23 and 33 wk gestation $(p<0.03)$.

Plasma GSII concentrations. There were no significant differences in GSH concentrations among the three groups of infants (Table 2). Least squares linear regression analysis of GSH as a function of gestational age, however, gave a line with a slope of $0.06 \mu \mathrm{M} \mathrm{GSH} /$ wk gestation (Fig. 3) that was different from $0(p$ $<0.004)$. Furthermore, when infants less than 34 wk were compared with those more than 34 wk (Table 3), arterial plasma GSH concentrations were higher in the older infants $(p<0.006)$.

Simultaneous measurements of arterial and venous plasma GSH concentrations revealed that the arterial GSH concentrations were roughly $10 \%$ less than the corresponding venous concentrations. By paired $t$ test, this difference was statistically significant for the group as a whole $(p<0.02)$ and for the group between 23 and 33 wk gestation $(p<0.02)$.

Plasma GSH/GSSG ratio. Because plasma GSH concentra-

Table 1. Description of patient population*

\begin{tabular}{ccccc}
\hline Group & $\begin{array}{c}\text { Number } \\
\text { of } \\
\text { patients }\end{array}$ & $\begin{array}{c}\text { Gestational } \\
\text { age } \\
(\mathrm{wk})\end{array}$ & $\begin{array}{c}\text { Birth wt } \\
(\mathrm{kg})\end{array}$ & $\begin{array}{c}\text { Number } \\
\text { of } \\
\text { samples }\end{array}$ \\
\hline 1 & 20 & $28.5 \pm 2.8$ & $1.05 \pm 0.26$ & 55 \\
2 & 43 & $29.9 \pm 4.1$ & $1.38 \pm 0.74$ & 131 \\
3 & 6 & $38.3 \pm 2.6$ & $3.09 \pm 0.32$ & 19 \\
Analysis of variance & & $p=0.001$ & $p=0.001$ & \\
Newman-Keuls test & & $3>2=1$ & $3>2=1$ & \\
\hline
\end{tabular}

* Subjects were recruited to the study and assigned to the appropriate group as described in Materials and Methods. All samples were obtained from indwelling umbilical artery catheters. Data are mean \pm SD. Data were analyzed statistically by one-way analysis of variance, for which the appropriate $p$ value is given, and further analyzed by Newman-Keuls test for multiple comparison, with differences at $p<0.05$ indicated. 
Table 2. GSH and GSSG concentrations in arterial plasma*

\begin{tabular}{cccccc}
\hline & & & UA plasma & UA plasma \\
Group & $\mathrm{FiO}_{2}$ & $\mathrm{PAO}_{2}(\mathrm{kPa})$ & $\mathrm{PaO}_{2}(\mathrm{kPa})$ & $\mathrm{GSH}(\mu \mathrm{M})$ & $\mathrm{GSSG}(\mu \mathrm{M})$ \\
\hline 1 & $0.25 \pm 0.07$ & $17.2 \pm 5.8$ & $9.8 \pm 2.5$ & $2.44 \pm 1.27$ & $0.82 \pm 0.55$ \\
2 & $0.52 \pm 0.30$ & $42.0 \pm 28.0$ & $8.8 \pm 2.6$ & $2.49 \pm 1.44$ & $0.63 \pm 0.48$ \\
3 & $0.58 \pm 0.33$ & $48.9 \pm 31.4$ & $8.1 \pm 3.3$ & $3.13 \pm 1.31$ & $0.44 \pm 0.44$ \\
Analysis of variance & $p=0.001$ & $p=0.001$ & $p=0.069$ & $p=0.293$ & $p=0.026$ \\
Newman-Keuls test & $3=2>1$ & $3=2>1$ & $\mathrm{NA}$ & $\mathrm{NA}$ & $1>2=3$ \\
\hline
\end{tabular}

* Plasma concentrations of GSH and GSSG were measured in samples from blood obtained from umbilical artery UA catheters as described in Materials and Methods. Values of $\mathrm{FiO}_{2}, \mathrm{PAO}_{2}$, and $\mathrm{PaO}_{2}$ were obtained from those measured as part of the normal clinical management of these patients and occurred before drawing blood samples. Data are mean \pm SD and are statistically evaluated by one-way analysis of variance and by Newman-Keuls multiple comparison test, with significant differences indicated at $p<0.05$. NA. data not different by one-way analysis of variance and therefore not tested by Newman-Keuls multiple comparison test.

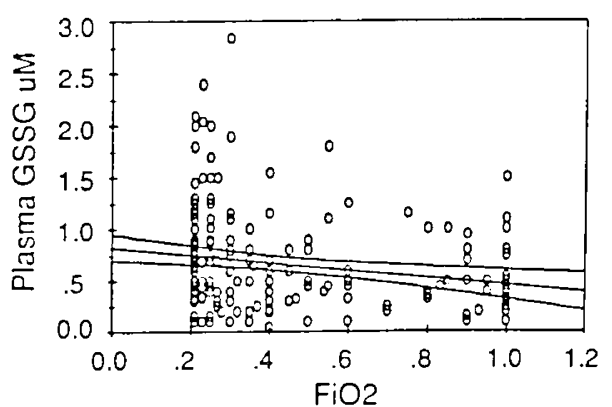

Fig. 1. We obtained 205 measurements of plasma GSSG $(\mu \mathrm{M})$ from 69 patients and plotted them is $\mathrm{FiO}_{2}$. The regression line is shown, with the $95 \%$ confidence intervals for the mean. The equation of the regression line is $y=-0.36 x+0.82$. The slope is significantly different from $0, p$ $<0.003$, with $r=0.21$.

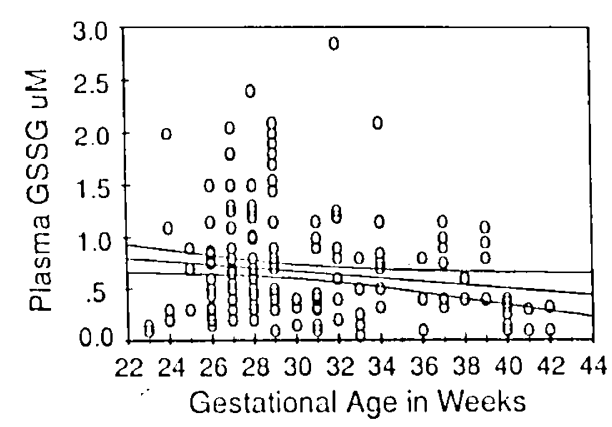

Fig. 2. We plotted the 205 measurements of plasma GSSG $(\mu \mathrm{M})$ vs gestational age in wk. The regression line is shown. with the $95 \%$ confidence intervals for the mean. The equation of the regression line is $y=-0.016 x+1.16$. The slope is significantly different from $0, p<0.03$, with $r=0.15$.

tions decreased with decreasing gestational age, we were concerned that GSH availability might be limiting the increase in GSSG noted with decreasing gestational age. Therefore, we performed least squares linear regression analyses of the GSH/GSSG ratios against $\mathrm{FiO}_{2}$ and gestational age. GSH/GSSG versus $\mathrm{FiO}_{2}$ gave a line with a slope that was not significantly different from 0 (data not shown), whereas GSH/GSSG versus gestational age (Fig. 4) gave a line with a slope of $0.66 / w k$ gestation that was different from $0(p<0.0001)$. Similarly, when infants of less than 34 wk gestational age were compared with those more than 34 wk (Table 3 ), plasma GSH/GSSG ratios were nearly twice as high in the older infants $(p<0.001)$.

\section{DISCUSSION}

Plasma GSSG concentrations. Plasma concentrations of GSSG were measurable in the infants studied and in most infants were greater than the 0.1 to $0.3 \mu \mathrm{M}$ typically observed in adult humans $(10,11)$. However, the plasma GSSG concentrations we measured were greatest in the infants exposed to the lowest oxygen tensions (Fig. 1). The group 1 infants, most of whom were ventilated with room air $\left(\mathrm{FiO}_{2}=0.21\right)$, were intended to serve as the control group for the group 2 infants, who were ventilated with elevated oxygen concentrations and in whom we intended to investigate the relationship between exposure to hyperoxia and plasma GSSG concentrations. However, we found greater arterial plasma GSSG concentrations in the group 1 infants than in the infants in groups 2 and 3 (Table 2). Furthermore, although the plot of plasma GSSG concentrations versus $\mathrm{FiO}_{2}$ showed considerable scatter, linear regression analysis revealed a statistically significant negative correlation between the two, $(p<$ 0.003 ) (Fig. 1). Obviously, this is a result we had not anticipated when we designed the study.

On the other hand, the correlation between gestational age and plasma GSSG concentrations (Fig. 2) is also significant and suggests that the degree of prematurity itself can be a significant determinant. In fact, one possible interpretation of the elevated plasma GSSG concentrations in group 1 infants, is that even room air $\left[\mathrm{FiO}_{2}=0.21, \mathrm{PO}_{2}=20.0-21.3 \mathrm{kPa}(150-160 \mathrm{~mm} \mathrm{Hg})\right]$ may constitute hyperoxia for very premature newborns. In retrospect, it is not illogical to find significant oxidant stress responses in a group of individuals who are exposed to partial pressures of oxygen several times the intrauterine oxygen tensions $\left[\mathrm{PO}_{2}=2.67-3.33 \mathrm{kPa}(20-25 \mathrm{~mm} \mathrm{Hg})\right]$ before they have completed the normal developmental progression of their antioxidant functions $(12,13)$.

Table 3. Comparison of infants by gestational age at birth*

\begin{tabular}{|c|c|c|c|c|c|c|}
\hline $\begin{array}{c}\text { Gestational age } \\
(\mathrm{wks})\end{array}$ & $\mathrm{FiO}_{2}$ & $\begin{array}{l}\mathrm{PAO}_{2} \\
(\mathrm{kPa})\end{array}$ & $\begin{array}{l}\mathrm{PaO}_{2} \\
(\mathrm{kPa}) \\
\end{array}$ & $\begin{array}{c}\text { GSSG } \\
(\mu \mathrm{M})\end{array}$ & $\begin{array}{l}\text { GSH } \\
(\mu \mathrm{M}) \\
\end{array}$ & GSH/GSSG \\
\hline $23-33$ & $0.39 \pm 0.26$ & $30.1 \pm 23.4$ & $9.1 \pm 2.3$ & $0.71 \pm 0.52$ & $2.40 \pm 1.38$ & $6.38 \pm 0.058$ \\
\hline $34-42$ & $0.69 \pm 0.32$ & $59.1 \pm 29.1$ & $8.6 \pm 3.7$ & $0.48 \pm 0.33$ & $3.44 \pm 2.12$ & $12.17 \pm 2.08$ \\
\hline Unpaired $t$ test & 6.776 & 7.009 & 1.182 & 2.696 & 3.930 & 3.750 \\
\hline$p$ & $<0.001$ & $<0.001$ & NS & 0.008 & $<0.001$ & $<0.001$ \\
\hline
\end{tabular}

* Data are mean \pm SD, with $t$ and $p$ (two tailed). There were 165 samples from 55 infants in the 23 to 33 wk group and 46 samples from 14 infants in the 34 to 42 wk group. 
Table 4. Arteriovenous differences in plasma GSH and GSSG concentrations*

\begin{tabular}{ccccccccc}
\hline $\begin{array}{c}\text { Gestational } \\
\text { age (wks) }\end{array}$ & $\begin{array}{c}\text { Arterial GSH } \\
(\mu \mathrm{M})\end{array}$ & $\begin{array}{c}\text { Venous GSH } \\
(\mu \mathrm{M})\end{array}$ & $t$ & $p$ & $\begin{array}{c}\text { Arterial GSSG } \\
(\mu \mathrm{M})\end{array}$ & $\begin{array}{c}\text { Venous GSSG } \\
(\mu \mathrm{M})\end{array}$ & $t$ \\
\hline $23-33$ & $2.10 \pm 0.17$ & $2.42 \pm 0.19$ & 2.635 & 0.011 & $0.73 \pm 0.07$ & $0.61 \pm 0.06$ & 2.277 & 0.027 \\
$34-42$ & $3.45 \pm 0.32$ & $3.54 \pm 0.43$ & -0.332 & 0.747 & $0.29 \pm 0.06$ & $0.17 \pm 0.03$ & 1.848 & 0.095 \\
$23-42$ & $2.32 \pm 0.16$ & $2.61 \pm 0.18$ & 2.539 & 0.014 & $0.66 \pm 0.06$ & $0.53 \pm 0.05$ & 2.644 & 0.010 \\
\hline
\end{tabular}

* A total of 66 samples were analyzed: 55 were from the 23 to 33 wk gestation infants: 11 were from the $\geq 34$ wk gestation infants. Data are mean \pm SD. Arterial and venous GSH and GSSG measurements were compared using paired $t$ test with $t$ and $p$ (two tailed). Arterial samples were obtained from the indwelling umbilical artery catheter; venous samples were obtained from indwelling umbilical venous catheters with their tips in the right atrium.

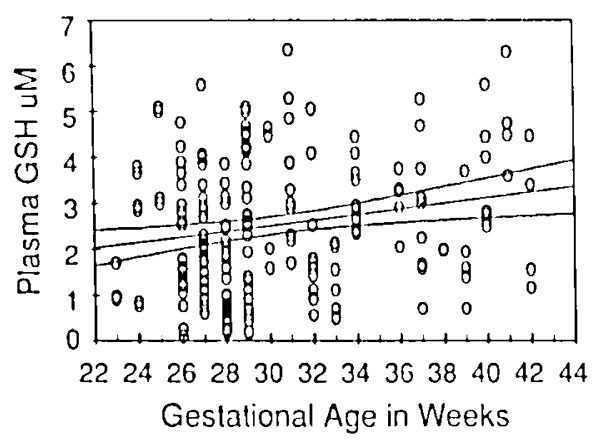

Fig. 3. We obtained 205 measurements of plasma GSH $(\mu \mathrm{M})$ from 69 patients and plotted them is gestational age in wk. The regression line is shown, with the $95 \%$ confidence intervals for the mean. The equation of the regression line is $y=0.06 x+0.70$. The slope is significantly different from $0, p<0.004$, with $r=0.20$.

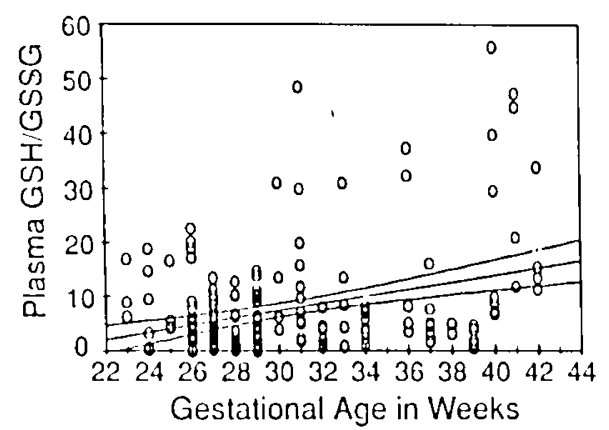

Fig. 4. We calculated the GSH/GSSG ratios for all 205 measurements and plotted them is gestational age in wk. The regression line is shown. with the $95 \%$ confidence intervals for the mean. The equation of the regression line is $y=0.66 x-12.4$. The slope is significantly different from $0, p<0.0001$, with $r=0.32$.

If observed in properly matched groups of experimental animals, the differences in GSSG concentrations that we observed between these infants and normal adults would be interpreted as evidence of marked oxidant stress in the experimental group. However, comparisons of the infants with healthy adults are not as readily interpretable. The data do suggest that these infants may not be able to deal adequately with the oxidant stresses they are encountering. Elevated plasma GSSG concentrations could result if the infants were exposed to oxidant stresses that were excessive compared with normal adults, if infants' antioxidant enzyme activities were compromised, or some combination of both. Interestingly, the plasma GSSG concentrations we observed in the prematurely born infants also are more than the GSSG concentrations observed by Buhl et al. (10) in HIV-infected adults or by us in infected children (11), despite the extensive evidence implicating the involvement of oxidant stress mechanisms in the progression of HIV infection (14).

In infants with both arterial and venous catheters, we found that GSSG concentrations in arterial plasma were significantly greater than simultaneously determined GSSG concentrations in venous plasma (Table 4). This suggests a measurable net release of GSSG by the lungs. Similar arteriovenous differences in GSSG concentrations across the lungs have been reported for rats treated with the redox-cycling lung toxin, paraquat (1). Not all individual GSSG differences were negative, probably reflecting relatively small effects of the lungs on plasma GSSG, often close to the limitations of the precision of our analytical methods. In addition, GSSG has been shown to support GSH synthesis in isolated rat lungs, albeit less efficiently than with GSH (15), and in individual patients it is possible that GSSG released from other organs could have been undergoing net uptake by the lungs.

The clinical and physiologic implications of the GSSG concentrations observed in these infants are not clear. Presently, we are unaware of any evidence that elevated plasma concentrations of GSSG mediate any adverse responses. Thiol-disulfide exchange with protein thiols by GSSG can modulate the activities of certain enzymes (16), but the physiologic or toxicologic effects of GSSG are less certain than are the implications that increased rates of efflux of GSSG reflect increased exposure to oxidants such as hydrogen peroxide, compromise of cellular glutathione reductase activities, or both.

Plasma GSH concentrations. The plasma concentrations of GSH in the infants in the present study are lower than the 6 to $10 \mu \mathrm{M}$ concentrations we typically observe in plasma from healthy adult humans. Although the comparison of the GSH concentrations in these infants with the corresponding values in healthy adults is subject to the same limitations previously discussed for GSSG, the comparison does offer a useful perspective to view the data on the infants. In the infants we studied, there was a tendency for the older infants to exhibit higher plasma GSH concentrations, and a linear regression analysis of the data shows a statistically significant $(p<0.036)$ correlation between GSH and gestational age at birth (Fig. 3). As with plasma GSSG, considerable scatter is seen in the GSH concentrations, but also as with GSSG, these plasma GSH concentrations are distinctly different from the concentrations we typically observe in healthy adults. In fact, the plasma GSH concentrations observed in the infants are comparable to the GSH concentrations found in HIV-infected adults and children $(10,11)$.

The importance of GSH in normal functioning of cellular defense mechanisms is well recognized, and depletion of GSH by fasting, restricting dietary sources of divalent sulfur or treatment with L-buthionine-[S,R]-sulfoximine has been reported to enhance the sensitivity of experimental animals to the adverse effects of hyperoxia (17-20) and other potentially damaging challenges such as has been characterized for toxic doses of acetaminophen (21). In addition to the stresses created by increased oxidation of GSH to GSSG, the hyperoxia-stimulated peroxidation of endogenous lipids and subsequent reactions produce several electrophilic products, such as 4-hydroxy-2-nonenal that can irreversibly consume GSH via Michacl addition reactions and exert cytotoxic effects if GSH availability is insufficient for adequate cellular defense functions (22).

GSH concentrations of lung tissue and extracellular fluids (plasma, alveolar lining fluid) decrease in experimental animals 
exposed to hyperoxia $(7,23-24)$, but the extents of depletion of GSH observed in these studies are moderate relative to the changes in GSH observed in the models of acute lethal injury such as acetaminophen poisoning, where more marked GSH depletion thresholds to toxicity are observed (21). The moderate depletion of whole tissue GSH observed suggests that the critical targets for hyperoxic lung injury may lie in specific cell types or subcellular pools in which depletion is not evident through analysis of whole tissue, plasma, or epithelial lining fluids. The reports that long-term administration of L-buthionine-[S,R]-sulfoximine to mice (25) and newborn rats (26) causes adverse effects that in some ways mimic those of hyperoxic exposure lend further credibility to the hypothesis that localized depletion of GSH can be a significant determinant of hyperoxic injury. It follows that the inability to sustain suitable supplies of GSH can contribute significantly to the damaging effects of hyperoxia. Although the primary function of plasma GSH may be to serve as a cysteine supply for GSH and protein synthesis by extrahepatic tissues $(1,21)$, more recent studies report a dramatic stabilization of nitric oxide in a bioactive form by adduct formation with GSH (27). The idea that plasma GSH status might appreciably affect the disposition and effects of nitric oxide produced by vascular endothelial cells is attractive but requires further investigation. The plasma GSH concentrations we observed in the prernature infants suggest a relative deficiency wherein some form of supplementation might benefit some patients. The significantly greater concentrations of GSH in the plasma of the older infants (Table 3, Fig. 4) suggest developmental effects, but whether increased synthesis and efflux or decreased clearance from the vascular compartment with increasing gestational age is responsible is not known.

Purucker and Wernze (28) have recently reported measurements of plasma GSH and GSSG in blood drawn from different vascular beds in 10 patients without liver dysfunction and concluded that substantial translocation of GSH from the liver to the plasma did not occur in humans. These investigators estimated plasma GSH concentrations to be uniformly around 1 $\mu \mathrm{M}$. However, they did not indicate the use of inhibitors of $\gamma$ GGT in the workup of the blood samples they obtained, whereas we added the blood to equal volumes of ice-cold buffer containing $50 \mathrm{mM}$ serine borate immediately on obtaining the blood. Serine borate inhibits GGT efficiently and quickly, and although we did not measure plasma or serum GGT activities in the infants in our study, GGT activities typically are higher in newborn infants $(10$ to $100 \mathrm{U} / \mathrm{L}$ ) than in adults (up to $30 \mathrm{U} / \mathrm{L}$ ) (29). Purucker and Wernze did measure GGT activities in their subjects and found $14.9 \pm 2.6 \mathrm{U} / \mathrm{L}$. This means that, on average, $1 \mathrm{~L}$ of their subjects' plasmas had enough GGT activity to degrade $14.9 \mu \mathrm{mol}$ of $\gamma$-glutamyl-containing substrate in 1 min at $25^{\circ} \mathrm{C}$, which is far in excess of the GSH concentrations we find even in adults. It appears that in their study about $10 \mathrm{~min}$ elapsed from drawing the blood to deactivation of GGT activity by addition of sulfosalicylic acid to denature plasma proteins. They report very high analytical recoveries of added GSH and GSSG, which may indicate that other factors, such as availability of acceptor substrate, could control GGT-mediated conversions in nonproportional ways. The GSH concentrations we determined with monobromobimane and reverse-phase HPLC are in good agreement with the concentrations we estimated with the enzyme recycling methods.

The relatively low GSH concentrations we observed in the infants couple with the relatively high concentrations of GSSG to result in GSH/GSSG ratios (Fig. 4) that are very low, relative to the ratios we find in adults (20-100). Insofar as this ratio offers a useful assessment of oxidant stress status (5), these infants are uniformly under an oxidant stress that decreases with gestational maturation.

In infants with both arterial and venous catheters, we found that GSH measurements in arterial plasma were significantly lower than simultaneous measurements in venous plasma (Table
4). The negative arteriovenous difference is consistent with a net uptake of GSH by the lungs. Again, not all individual GSH differences are negative, which could result from attempts to measure biologic differences that are close to the error of our analytical methods. However, net pulmonary release of GSH secondary to cell lysis could also account for a real net release of GSH to the circulation of some individuals.

Conclusion. In summary, we found that plasma GSSG concentrations were elevated in preterm infants compared with adults and that this elevation was inversely correlated with gestational age. The positive gradient for GSSG across the lung suggests that at least some plasma GSSG is derived from lung tissue. This increase in GSSG could be the result of increased production of reactive oxygen species, deficient antioxidant activities, or some combination of both. Additionally, we found that plasma GSH concentrations were relatively low in preterm infants compared with normal adults and that GSH concentrations increased with increasing gestational age. Whether this apparent deficiency leaves these infants more susceptible to tissue injury by reactive oxygen remains to be determined. Finally, we found a negative gradient for GSH across the lung, suggesting lung uptake of GSH. To our knowledge, this is the first report of evidence for GSH uptake by the lung in humans.

\section{REFERENCES}

1. Adams Jr JD, Lauterburg BH, Mitchell JR 1983 Plasma glutathione and glutathione disulfide in the rat: regulation and response to oxidative stress. J Pharmacol Exp Ther 227:749-754

2. Lauterburg BH, Smith CV. Hughes H. Mitchell JR 1984 Biliary excretion of glutathione and glutathione disulfide in the rat: regulation and response to oxidative stress. J Clin Invest 73:124-133

3. Jenkinson SG, Spence TH, Lawrence RA, Hill KE, Duncan CA. Johnson KH 1987 Rat lung glutathione release: response to oxidative stress and selenium deficiency. J Appl Physiol 62:55-60

4. White CW, Mimmack RF, Repine JE 1986 Accumulation of lung tissue oxidized glutathione (GSSG) as a marker of oxidant induced lung injury. Chest 89:111S-113S

5. Smith CV 1991 Correlations and apparent contradictions in assessment of oxidant stress status in vivo. Free Radic Biol Med 10:217-224

6. Hazinski TA, Kennedy KA, France ML, Hansen TN 1988 Pulmonary $\mathrm{O}_{2}$ toxicity in lambs: physiological and biochemical effects of endotoxin infusion. J Appl Physiol 65:1579-1585

7. Hansen TN, Smith CV, Gest AL, Smith HW, Giesler M 1990 Biochemical manifestations of oxygen toxicity in the newborn lamb. Pediatr Res 28:613617

8. Wispé JR, Roberts RJ 1987 Molecular basis of pulmonary oxygen toxicity. Clin Perinatol 14:651-666

9. Zar JH 1984 Biostatistical Analysis, 2nd Ed. Prentice-Hall, Englewood Cliffs, NJ

10. Buhl R, Holroyd KJ, Mastrangeli A, Cantin AM, Jaffe HA, Wells FB, Saltini C. Crystal RG 1989 Systemic glutathione deficiency in symptom-free HIVseropositive individuals. Lancet 2:1294-1298

11. Smith CV. Hansen TN. Hanson IC, Shearer WT 1990 Glutathione concentrations in plasma and blood are markedly decreased in HIV-infected children. Sixth International Conference on AIDS. San Francisco, 2:368

12. Frank L, Sosenko IRS 1987 Prenatal development of lung antioxidant enzymes in four species. J Pediatr 110:106-110

13. Frank L 1991 Developmental aspects of experimental pulmonary oxygen toxicity. Free Radic Biol Med 11:463-494

14. Staal FJT, Ela SW, Roederer M, Anderson MT, Herzenberg LA 1992 Glutathione deficiency and human immunodeficiency virus infection. Lancet 339:909-912

15. Berggren M, Dawson J, Moldeus P 1984 Glutathione biosynthesis in the isolated perfused rat lung: utilization of extracellular glutathione. FEBS Lett 176:189-192

16. Gilbert HF 1990 Molecular and cellular aspects of thiol/disulfide exchange. Adv Enzymol 63:69-172

17. Deneke SM, Lynch BA, Fanburg BL 1985 Transient depletion of lung glutathione by diethylmalcate enhances oxygen toxicity. J Appl Physiol 58:571574

18. Deneke SM, Lynch BA. Fanburg BL 1985 Effects of low protein diets or feed restriction on rat lung glutathione and oxygen toxicity. J Nutr 115:726-732

19. Deneke SM, Steiger V, Fanburg BL 1987 Effect of hyperoxia on glutathione levels and glutamic acid uptake in endothelial cells. J Appl Physiol 63:19661971

20. Smith LJ, Anderson J, Shamsuddin M, Hsuch W 1990 Effect of fasting on 
hyperoxic lung injury in mice. The role of glutathione. Am Rev Respir Dis 141:141-149

21. Smith CV, Mitchell JR 1989 Pharmacological aspects of glutathione in drug metabolism. In: Dolphin D, Poulson R, Avramovic O (eds) Coenzymes and Cofactors. John Wiley \& Sons, New York, pp $1-44$

22. Spitz DR, Sullivan SJ, Malcolm RR, Roberts RJ 1991 Glutathione dependent metabolism and detoxification of 4-hydroxy-2-nonenal. Free Radic Biol Med $11: 415-423$

23. Jenkinson SG, Black RD, Lawrence RA 1988 Glutathione concentrations in rat lung bronchoalveolar lavage fluid: effects of hyperoxia. J Lab Clin Med 112:345-351

24. Stenzel JD, Welty SE, Benzick AE, Smith CV, Hansen TN 1993 Hyperoxic lung injury in Fischer-344 and Sprague-Dawley rats in vivo. Free Radic Biol Med 15:531-539
25. Martensson J, Jain A, Frayer W, Meister A 1989 Glutathione metabolism in the lung: inhibition of its synthesis leads to lamellar body and mitochondrial defects. Proc Natl Acad Sci USA 86:5296-5300

26. Martensson J, Jain A, Stole E, Frayer W, Auld PAM, Neister A 1991 Inhibition of glutathione synthesis in the newborn rat: a model for endogenously produced oxidative stress. Proc Natl Acad Sci USA 88:9360-9364

27. Clancy RM, Abramson SB 1992 Novel synthesis of s-nitrosoglutathione and degradation by human neutrophils. Anal Biochem 204:365-371

28. Purucker E, Wernze H 1990 Hepatic efflux and renal extraction of plasma glutathione: marked differences between healthy subjects and the rat. Klin Wochenschr 68:1008-1012

29. Bergmeyer HU 1974 Methods of Enzymatic Analysis. 2nd Ed. Academic Press, New York, pp 715-718 